\title{
Monument Des Martyrs Dans La Ville d'Abidjan : Entre Logique Urbaine Et Légitimation Politique (2002 À 2010)
}

\author{
Barnabé Cossi Houédin \\ N'Guessan Daniel Djédjé \\ Université Félix Houphouët-Boigny d'Abidjan/ Côte d'Ivoire \\ Institut d'Ethno-Sociologie (IES) \\ Tata Mariam Fofana \\ Département de Sociologie et d'Anthropologie \\ Université Lorougnon Guédé de Daloa, Côte d'Ivoire
}

doi: 10.19044/esj.2017.v13n25p207 URL:http://dx.doi.org/10.19044/esj.2017.v13n25p207

\begin{abstract}
This text handles with the fate of the built monuments to the memory of victims built as martyrs in a context of crisis in Côte d'Ivoire. He is mainly interested in the way the political systems legitimize them since their construction. As regards him "Monument of the martyrs", the results show a kind of legitimization of the power in sync with the mobilization of urban logics. Which passes by the gratitude and the valuation to the deaths set up as martyrs and by the strategies of identification, resistance and identity domination. The article tends to highlight a variability of the fields of use of the monument as social resource and the representations which are associated with it, the links built between such representations and the arrangement of the city during period going from 2002 till 2010. As a matter of fact, the text shows that the legitimization of the monument in the urban dynamics favors rationalities the coherence of which strengthens the public action regarding governance, regarding production of a shape of citizenship, a type of collective identity and spatial marking
\end{abstract}

Keyswords: Identity ; Martyr ; Memory ; Monument ; Governance

Résumé

Ce texte traite du sort des monuments bâtis à la mémoire de victimes construites comme des martyrs dans un contexte de crise en Côte d'Ivoire. Il s'intéresse principalement à la façon dont les régimes politiques les légitiment depuis leur construction. En ce qui concerne le « monument des martyrs ", les résultats montrent une sorte de légitimation 
du pouvoir en phase avec la mobilisation de logiques urbaines. Ce qui passe par la reconnaissance et la valorisation aux morts érigés en martyrs et par les stratégies d'identification, de résistance et de domination identitaire. L'article tend à mettre en évidence une variabilité des champs d'usage du monument comme ressource sociale et les représentations qui y sont associées, les liens construits entre de telles représentations et l'aménagement de la ville au cours de la période allant de 2002 à 2010. En somme, le texte montre que la légitimation du monument dans les dynamiques urbaines favorise des rationalités dont la cohérence consolide l'action publique en matière de gouvernance, de production d'une forme de citoyenneté, d'un type d'identité collective et de marquage spatial.

Mots-clés: Identité ; Martyr ; Mémoire ; Monument ; Gouvernance

\section{Introduction}

Le «monument des martyrs » a été inauguré le 24 janvier 2002 dans la ville d'Abidjan en Côte d'Ivoire. Il aurait été édifié suivant les ordonnances du Président Gbagbo Laurent en vue de commémorer les victimes ${ }^{10}$ liées aux évènements de son accession à la présidence de la République après les élections d'octobre 2000 (Sivori 2000 ; Tiesse, 2000; Gbato, 2002). Présenté par ses promoteurs (le Président Gbagbo Laurent et les militants ou sympathisants du FPI ${ }^{11}$ ) comme un monument commémoratif, il aurait acquis un statut particulier du fait que son édification a été motivée par la volonté de reconnaissance de ce nouveau chef d'Etat vis-à-vis du « peuple » qui

10 L'édification du monument aurait été initialement incitée pour rendre hommage aux victimes des évènements des 24,25 et 26 octobre 2000 où, suite à l'appel de Gbagbo Laurent, le " peuple » est descendu dans les rues pour contester ce qu'il qualifiait de braquage électoral opéré par le candidat sortant, le Général Robert Guéi. Ensuite, à défaut de la volonté de construire de nouveaux monuments en hommage des victimes de " la tentative de coup d'Etat du 19 septembre 2002 ", des " massacres " relatifs aux évènements des 14, 15, 16 novembre 2004 à l'Hôtel Ivoire (Laquachenn, 2005), l'identité des victimes, qu'elles soient des figures emblématiques de la scène politique ou pas, a été aussi associée à ce monument. Par la suite, en raison de la continuité de la crise et l'implication de présupposés acteurs externes tel que la France (l'ancien colonisateur) (Koulibaly, 2003), il a été surtout brandi par les militants ou sympathisants du FPI comme la symbolique de toutes les victimes de la période de crise allant de l'année 2000 à 2010.

11 Front Populaire Ivoirien, parti politique dont est issu l'ex-président de la République Gbagbo Laurent. Ce dernier est resté au pouvoir de 2000 à 2010 et le parti dont il est issu, le FPI était considéré à cette période comme le parti au pouvoir et l'un des partis politiques significatifs de la scène politique en Côte d' Ivoire 
l'a soutenu et a participé à le positionner à ce rang. Ainsi, ses conditions d'édification auraient été facilitées par l'État avec plus ou moins les fonctions premières de contribuer, au plan institutionnel ${ }^{12}$, à honorer et préserver l'identité desdites victimes. De plus, selon les objectifs assignés par le Président Gbagbo Laurent et les militants du FPI, il a été le signe ou le point de repère commun entre le " peuple » et l'Etat. En tant que tel, il est supposé faire figure d'un modèle d'infrastructure permettant de traduire non seulement une légitimation du pouvoir et un support à la citoyenneté mais aussi, faire émerger aux plans local et national, l'idée d'une identification concertée à travers laquelle l'espace urbain abidjanais serait à l'épreuve d'une politique publique de cohésion sociale.

Mais, sur la base de ses conditions d'émergence et la variété des objectifs susmentionnés, cet investissement mémoriel est resté marqué par l'identité de ces derniers et passerait aux yeux de leur principal adversaire politique ${ }^{13}$ comme un investissement dont les motivations ou les conditions d'édification sont problématiques. Sous ce rapport, le monument a donc progressivement été inscrit dans les rivalités politiques entre le FPI et le RDR. Depuis donc son édification jusqu'à son état actuel de ruines ${ }^{14}$, l'objectif (réel ou supposé) qui lui est attribué, celui de contribuer à la commémoration de martyrs dits nationaux, est allé de pair avec les controverses entre ces deux familles politiques. Ce qui fait du monument un élément essentiel dans la lecture des conflits du même champ dans la ville d'Abidjan. A preuve, sa forme initiale et ses présentes ruines ( voir photo ci-après) sont d'une part deux positions présentées dans la ville d'Abidjan comme les symboles du décalage permanent entre les perceptions, la revendication et l'usage concurrents des victimes dont elles sont

${ }^{12} \mathrm{Au}$ cours de la journée de « deuil national et de commémoration des martyrs » organisée le 09 Novembre 2000, Gbagbo Laurent fit les promesses suivantes : i) prendre un décret consacrant tous les 24 octobre, journée des martyrs sur toute l'étendue du territoire, ii) baptiser l'une des principales voies de la ville de " boulevard de martyrs » et enfin, iii) construire un mémorial de martyrs en l'honneur des victimes des évènements du 24, 25 et 26 octobre 2000. Lire (Pépé, 2000)

${ }^{13}$ Il s'agit du RDR: Rassemblement Des Républicains, un autre parti politique significatif de la scène politique ivoirienne

${ }^{14}$ L'enquête a révélé que le monument a d'abord été l'objet d'une première attaque le 24 mars 2004 lors de la marche organisée par les militants du RDR et du PDCI pour protester contre certaines pratiques du gouvernement qu'ils désignent de dictature de Gbagbo Laurent et du FPI, parti au pouvoir. Les troubles générés par cette marche interdite ont débouché sur une première « profanation »et dégradation du monument. Ensuite, avec la crise post-électorale de 2010, sa destruction a été achevée, laissant à l'observation les restes de sa fondation encore présente sur le site où il a été édifié 
censées être représentatives ou non pour les deux partis politiques . Ces mêmes états du monument seraient d'autre part affichées dans l'imaginaire de la ville pour révéler l'écart entre le travail d'histoire et de mémoire respectivement diffusé par les mêmes familles politiques et qui les accompagnent.

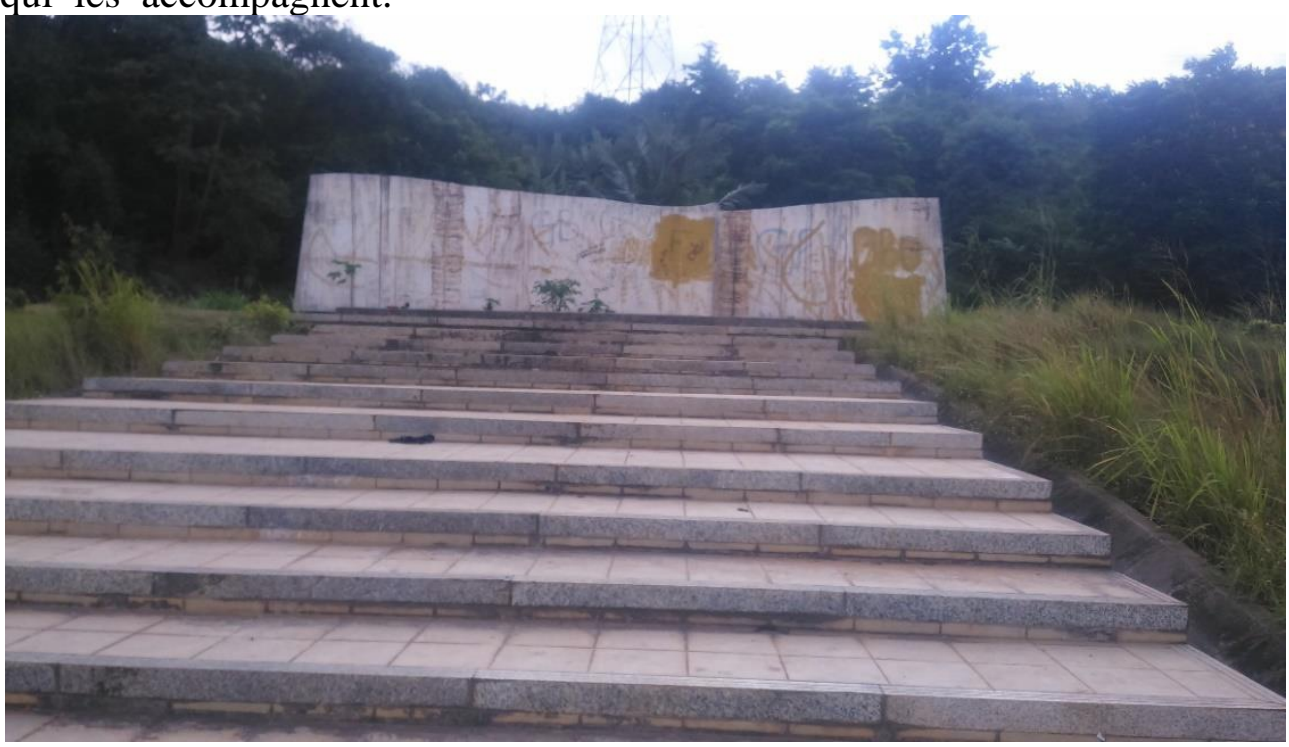

Source : Enquête de terrain, Houédin, 2015.

Photo : Aperçu des ruines du «monument des martyrs » :après la destruction du monument, il ne reste plus que le socle qui lui servait de fondation

D'un côté, depuis son édification, le Président Gbagbo Laurent et son régime ${ }^{15}$, à travers la politique de "refondation culturelle » (Dépri, 2001) ont entrepris de l'exploiter à des fins opérationnelles. En effet, transformant la victoire électorale et le caractère national conféré au monument en normes morales ou institutionnelles pour justifier son édification, ces derniers ont eux-mêmes construit, entretenu et répandu, aussi bien au niveau local que national, le succès politique dont cette

15 Doter la ville en infrastructures telles que les monuments aurait été parmi les priorités du Gouverneur du District d' Abidjan Djédji Amondji Pierre, l'un des acteurs de premier rang du FPI. A l'examen de ces propos de campagnes recueillis par Téha et Gbapo (2002), il entendait construire des monuments dans la ville d'Abidjan s'il était confirmé au poste de Gouverneur dudit District Au cours de l'enquête, certains interviewés ont révélé que c'est ce qu'il aurait fait en s'impliquant de façon active dans l'édification du " monument des martyrs » avec l'appui du Ministère de la construction et de l'urbanisme comme chef d'œuvre. Enfin, après inauguration du monument, sa gestion aurait été confiée au district d'Abidjan, institution dont il avait la charge de gouverner. 
œuvre d'art ${ }^{16}$ restait le symbole. De l'autre côté, pour Alassane Ouattara $^{17}$ et les siens du RDR, cette approche du monument serait plutôt un échec. Elle tend, semble-t-il, à masquer la réalité sur l'identité des martyrs célébrés . Et, par-delà ce fait, les efforts significatifs que ce leader du RDR et ces militants mènent non seulement en matière de lutte politique mais aussi, dans la construction de l'histoire de leur parti (Houédin et N'Guessan, 2017).

Investissement mémoriel mais aussi patrimoine culturel permettant d'inscrire lesdits martyrs dans la mémoire collective de la ville, cette infrastructure publique est de fait l'objet de débat entre les militants et sympathisants de ces partis rivaux. En effet, les fonctions officielles d'infrastructure « neutre » servant à l'embellissement de la ville attribuées par le Président Gbagbo Laurent et les sympathisants du FPI ne sont pas partagées par le RDR et son leader Alassane Ouattara. Et, dans un tel contexte (social et politique), il est devenu l'objet de fréquentes contestations et de méfiance de la part du second parti. L'enquête exploratoire permet de rendre compte du désintérêt de cette nouvelle catégorie d'acteurs au pouvoir depuis 2010, vis-à-vis des ruines du monument. Désintérêt d'ailleurs accentué par une volonté manifeste de le laisser sous silence dans la politique de rénovation urbaine enclenchée après la crise postélectorale en 2011 (Houédin et N'Guessan, 2017). Pourtant, les militants du FPI s'attachent toujours à ce que l'histoire associée à de telles ruines ne soit pas détournée de celle diffusée à travers les caractéristiques initiales du monument.

Le saisissant ainsi comme monument commémoratif (Choay, 1992 ; Bruneau et Papoulidis, 2003) en passant par la production de liens réels ou supposés entre l'histoire de ces partis politiques et son existence dans la ville d'Abidjan, de façon réciproque, les militants du RDR et du FPI défendent leurs positions vis-à-vis de l'évolution de son statut. Bâti à l'origine pour être un actif à inscrire dans la mémoire de la ville, il est devenu l'objet de deux positions contradictoires : d'une part, les sympathisants d'Alassane Ouattara en tant que ceux qui s'opposent à cette existence et d'autre part les sympathisants de Gbagbo Laurent en tant que ceux qui l'ont toujours

\footnotetext{
${ }^{16} \mathrm{~A}$ titre de rappel, Houédin et N'Guessan (2017) dans une précédente publication relative aux monuments dans la ville d'Abidjan désignaient principalement sous ce concept les monuments sculptés. Pour ce qui est du "monument des martyrs ", cette appellation totalisante est symbolique en ce sens qu'elle permet de mettre en relief le lien entre le caractère dit esthétique du monument à ses objectifs d'embellissement urbain et son statut de monument engagé

${ }^{17}$ Cet acteur est connu pour être le principal leader du RDR. Entre 2000 et 2010, il était encore dans l'opposition.
} 
soutenu. De façon conséquente, même dans son état de ruines, il est devenu un objet d'identification et de desidentification au-delà duquel se révèlent aussi les alliances et rivalités entre les groupements politiques significatifs du pays (Assouman, 2015).

Bien plus, prenant appui sur le décalage de position autour du monument ou de ses ruines, ce n'est pas une erreur d'affirmer qu'il a été affiché à un moment donné comme un avantage identitaire pour Gbagbo Laurent et les militants du FPI . Il en est de même de sa perception par les partis de l'opposition de l'époque (2002-2010) comme un symbole identificatoire visant à défavoriser le RDR et son leader Alassane Ouattara dans les compétitions politiques. En dépit donc de la méfiance qui a émaillé son existence, des formes de contestations politiques dont il a fait l'objet et de l'influence du changement politique qu'il a subie, les motivations sociales par lesquelles le « monument des martyrs » a été promu restent toujours d'actualité chez les sympathisants de Gbagbo Laurent.

Ces remarques offrent un éclairage sur l'intérêt de cette étude sur le «monument des martyrs» dans la ville d'Abidjan. Elles contribuent ainsi à mettre en exergue d'autres paramètres sociaux encore susceptibles de renseigner sur l'importance des transformations identitaires ou urbaines associées à l'existence dudit monument. En premier, il y a le fait que le «monument des martyrs » soit perçu par les militants du RDR comme érigé uniquement en l'honneur des martyrs du FPI ou des sympathisants de Gbagbo Laurent. Dans ce sens, des monuments comme « le monument de la liberté » (Yopougon), " le monument des éléphants dont les trompes se coupent » (PortBouet), " le monument les Mille Générations » (Plateau) et encore le «monument du rond-point de Cocody- Saint Jean », symbolisant la rupture des liens de l'esclavage ou de la servitude, ont été édifiés sous l'idéologie du nationalisme et détruits sous le prétexte $\mathrm{du}$ lien particulier avec l'idéologie de l'ancien pouvoir à partir de 2011 (Houédin et N'Guessan, 2017).

Deuxièmement, la partie Sud du « Boulevard Latrille ${ }^{18}$ a été rebaptisée « boulevard des martyrs » par le pouvoir du FPI en 2000. L'image de cette partie du boulevard a été renforcée avec les évènements des 14, 15, 16 novembre 2004 à l'Hôtel Ivoire. A la prise $\mathrm{du}$ pouvoir par Alassane Ouattara en 2010, 1' ancienne appellation de cette même partie du boulevard a été relégitimée .Troisièmement, il

${ }^{18}$ Ce boulevard situé dans la commune de Cocody, l'une des communes de la ville d'Abidjan, aurait été baptisé il y a plus d'une cinquantaine d'années en l'honneur d'André Latrille. Ce dernier fut Gouverneur de la colonie de Côte d'Ivoire de 1843 à 1845 
existe un autre monument des martyrs qui est entretenu par le pouvoir d'Alassane Ouattara ${ }^{19}$. Il est amovible et apparait dans la commune $\mathrm{d}^{\prime} \mathrm{Abobo}^{20}$ lors de commémoration d'évènements dits nationaux ${ }^{21}$. Ces différents constats rendent pertinents une analyse des logiques qui circulent ou s'affrontent autour du monument. Dans cet article, l'accent est plutôt mis sur la façon dont le pouvoir de Gbagbo Laurent a construit la légitimité du monument et la manière dont les sympathisants du FPI se représentaient sa présence dans la ville d'Abidjan. Partant de ce fait, la question qui oriente cette étude est la suivante : Comment le régime de Gbagbo Laurent a construit la légitimité du « monument des martyrs » dans la ville d'Abidjan ? De façon spécifique, quelles sont les représentations et les pratiques qui ont été générées autour de la présence du monument dans la ville d'Abidjan?

L'objectif de cette étude est de comprendre la façon dont le régime de Gbagbo Laurent a construit la légitimité du « monument des martyrs»» dans la ville d'Abidjan. Plus précisément, il est question d'examiner et de saisir le sens des représentations et des pratiques qui ont été générées par la présence d'un tel monument dans la ville d'Abidjan de 2002 à 2010 .

\section{Méthodologie}

L'article repose sur une enquête de terrain (Konan, 2015) menée au cours de l'année académique 2014-2015. Les principaux instruments de collecte de données sont le guide d'entretien et la grille d'observation d'Arborio (2007). Le corpus d'informations a été recueilli

\footnotetext{
${ }^{19}$ Depuis 2010, cet acteur de la scène politique est arrivé au pouvoir et occupe le statut de président de la République de Côte d'Ivoire

${ }^{20}$ Cette commune est l'une des dix communes de la ville d'Abidjan. Elle est communément désignée comme une base électorale du RDR.

${ }^{21}$ Il s'agit par exemple de la fête des lumières dans la ville d'Abidjan en fins d'années, de la commémoration des femmes martyres d'Abobo chaque 03 mars de l'année, cela, depuis la prise du pouvoir par Alassane Ouattara en 2010. Pour l'histoire, au cours de la crise post-électorale, une marche dite pacifique a été organisée dans la commune en question par les sympathisants d'Alassane Ouattara. Ils entendaient ainsi protester contre le maintien du pouvoir par le Président Gbagbo, le candidat sortant des élections présidentielles de 2010. Acte d'ailleurs qu'ils percevaient et désignaient comme une forme de confiscation du pouvoir, proclamant que ce dernier a perdu lesdites élections. Les troubles conséquents de cette marche ont débouché sur la mort, dit-on de sept (7) femmes, qui auraient été abattues par les forces de sécurité loyales au Président Gbagbo Laurent. C'est à partir de cet incident majeur de la crise post-électorale que du reste, le Conseil de sécurité de l'ONU (Organisation des Nations Unies) à voter le 30 mars 2011, la Résolution 1975 autorisant les soldats de ladite organisation et aux troupes françaises basées en Côte d'Ivoire de détruire les « armes lourdes » à la disposition du régime de Gbagbo Laurent.
} 
sur la base d'entretiens semi-directifs auprès d'acteurs d'institutions publiques $(n=6)$ telles que la Mairie d'Adjamé, le District d'Abidjan, les Ministères de la culture, de la construction et de l'urbanisme. A côté de la catégorie des experts issus de ces institutions publiques, l'enquête s'est aussi réalisée auprès de populations témoins $(n=9)$. A cet effet, cinq membres de syndicats d'étudiants ${ }^{22}$, quatre personnes communément appelées « titrologues» ${ }^{23}$ dans la commune d'Adjamé ont été sélectionnés de façon aléatoire et incluses dans l'étude. Le choix de ces derniers participants s'est fait sur la base des critères selon lesquels ils sont communément considérés comme des observateurs habituels de la vie politique et de l'intérêt qu'ils accordent à ce champ. Par ce statut, ils sont supposés être compétents à produire des discours plus ou moins fondés sur l'évolution les faits politiques aux niveaux local et national.

Même si les données recueillies à ce niveau demeurent peu précises et globalisantes, elles ont été confrontées au corpus d'informations collectées auprès de membres dits influents de la jeunesse, de conseillers et d'autres ${ }^{24}$ leaders d'opinion du RDR et du FPI $(n=10)$. Ont été respectivement interrogés, cinq membres dans chaque siège présentés comme suit : conseillers (2), responsables de jeunesse (2), responsable à charge de (X) (2), sympathisants de parti (4). Le choix de ces participants à l'étude est justifié par le fait que les militants de ces deux partis s'affichent dans la ville d'Abidjan comme ceux qui s'opposent au «monument des martyrs " pour le RDR ou le soutiennent au contraire s'agissant du FPI. Les échanges ont porté sur i) l'histoire de l'édification du monument, ii) la façon dont ces partis se représentent le monument avant et après sa destruction, iii) la façon dont ils justifient son édification, son état actuel de ruines, les enjeux autour de son état initial ou encore de son état de ruine mais également sur les statuts et les positions qui se

${ }^{22}$ Ces syndicats ont été pris en compte dans l'étude pour leurs probables liens avec les partis politiques en compétition. Lire à ce niveau Ibrahima (2017, p 383). Ils sont donc intégrés comme participants à l'étude à partir de l'idée selon laquelle ils ont la compétence de renseigner sur les pratiques politiques ou culturelles autour dudit monument

23 Selon Sandlar (2005, P. 229), les « « titrologues » sont des foules urbaines ivoiriennes qui, chaque matin, s'attroupent devant les kiosques à journaux et s'informent en parcourant les différentes unes. Les « titrologues » alimentent régulièrement un vaste réseau national de la rumeur par des informations qu'ils tirent des seules unes et qu'ils propagent comme des faits établis, des vérités. Commentaires et interprétations font le reste "

${ }^{24}$ Le statut réel de ces leaders d'opinion n'a plus ou moins pas pu être décliné au chercheur. 
jouent autour de ces états. La sélection des participants à l'étude a été essentiellement construite en fonction des différents objectifs spécifiques assignés à cette recherche et justifiée par les méthodes et techniques appropriées de l'approche qualitative (Pires, 1997 ; Chapoulie, 2000 ; Coté et Turgeon, 2002). Au total, un échantillon de 25 interviewés a été retenu et validé sur la base de la saturation des données (Pires, 1997).

La collecte des données a également permis de mobiliser des données secondaires issues de recherches documentaires, notamment les articles de presse écrite dite "indépendante » et celle dite proche du FPI ou du RDR. L'analyse de contenu ${ }^{25}$ (Mucchielli, 2006 ; Ghiglione, Landré, Bromberg et Molette, 1998) a été appliquée aux données collectées et retranscrites. Ce qui a permis d'aboutir aux résultats présentés dans ce travail.

\section{Résultats}

Les résultats de l'enquête permettent de souligner que les promoteurs du monument sont les partisans de Gbagbo Laurent. A partir du «monument des martyrs », ils tentent de mettre en place une identité urbaine à caractère nationale . Les propriétés de celle-ci se déclinent en plusieurs aspects dont les plus manifestes sont les cas cidessous examinés.

\section{De la mobilisation du concept de martyr dans la ville d'Abidjan : définition contextuelle et approche théorique}

Dans une perspective socio- anthropologique, Albert (2007) précise que le martyr est une forme de sécularisation de héros national, de victimes emblématiques d'une cause qualifiée de juste. Chez Bouzama et Campana (2015), la désignation de martyr se veut relative et contextuelle. Ces auteurs affirment d'ailleurs que l'identité de martyr se construit et se déconstruit à partir de contextes ou de positions sociales qui la déterminent ou dans lesquelles elle est promue. Pour ces derniers, la figure du martyr peut être soumise à une multiplicité de contextes et d'aspects qui la fabriquent et la font évoluer.

Dans le contexte ivoirien, le concept de martyr a été essentiellement mobilisé par le FPI et le RDR, deux partis politiques rivaux, pour désigner telles ou telles de leurs victimes au cours de la crise militaro-politique de 2000 à 2010. Considérées comme des «

25 L'accent a été mis sur l'analyse thématique en ce qui concerne le corpus issus des entretiens de terrain et l'analyse propositionnelle du discours tiré des extraits d'articles de la presse écrite. 
héros nationaux » ou des militants supposés être des modèles, ces victimes ont été inscrites à ce rang du fait de leur engagement « absolu » à défendre les valeurs de nationalisme, de patriotisme ou la diffusion des idéaux du parti politique dont elles sont issues. L'image dominante de ces dernières qui ressort du corpus de données recueillies montre essentiellement que, leur participation à la vie politique du parti et/ou du pays est valorisée par le risque plus ou moins mortel de ce choix (Albert, 2007). Rejoignant cette façon d'appréhender le martyr, le Président Gbagbo Laurent propose une définition de ceux célébrés au travers dudit monument en ces termes : "Ils sont tombés en combattant les mains nues sans haine... Dans les circonstances même de leur mort, ils ont indiqué le genre de vie qu'ils souhaitent pour l'humanité et pour les Ivoiriens. Ils ont fait de la démocratie une raison de mourir, c'est-à-dire une raison de vivre : offrir sa vie pour son pays, pour un idéal, pour un avenir meilleur ». (Extrait de discours recueilli par Sivori, 2000).

"... les vrais héros de notre histoire sont donc couchés, tombés morts. Les autres sont meurtris dans leur chair..." (Extrait du discours recueilli dans Fraternité Matin, 10 novembre 2000, p.4)

Outre ces traits dominants de la définition du martyr, dans cette étude, ce concept renvoie à une construction sociale de la valeur des victimes de la crise militaro-politique. Il permet aux leaders politiques qui en font usage d'associer de l'honneur et des significations valorisantes à l'image desdites victimes. Sa matérialité et son usage dans la ville d'Abidjan cacheraient un double système de légitimation et de delégitimation identitaire. Il rappelle la façon dont au plan politique, les dynamiques relatives à l'appropriation de l'espace urbain ainsi que les résistances à ces logiques permettent de comprendre la structuration ou les formes contemporaines du conflit social entre les partisans de Gbagbo Laurent et ceux d'Alassane Ouattara. C'est dans cette optique qu'il serait devenu un instrument stratégique aussi bien dans l'aménagement de l'espace urbain que dans les compétitions politiques locales.

En demeurant au centre des divergences mémorielles activées par les militants des deux leaders politiques susmentionnés, il constitue ainsi l'operateur à partir duquel les stratégies produites de part et d'autre passent d'un système d'action non concret à un système d'action concret. Sous un tel rapport, il devient ainsi l'outil par lequel les perspectives théoriques relatives aux travaux sur les monuments aux morts (Jagorel, 2014; Aubry et Matthieu de Oliveira, 2014) et la 
littérature sociologique sur la mémoire (Halbwachs, 1925, Haegel et Lavabre, 2010 ; Lavabre et Gensburger, 2012) sont rappelés. Ces travaux sont essentiels car, en servant de contrepoint ou d'inspiration aux pratiques mémorielles relevées dans le corpus de données, ils contribuent à préparer le cadre d'analyse ci-après et à comprendre les arguments développés dans le présent article.

L'article s'inscrit dans la perspective de l'action organisée (Crozier et Friedberg, 1977 ; Friedberg 1993). Sous un tel angle d'analyse, il permet de construire le lien entre les formes de légitimités suscitées et l'aménagement de la ville à cette époque en les logeant spécifiquement dans un système d'action précis. Dans cet ordre d'idées, l'accent est particulièrement mis sur les marges d'action limitées des acteurs qui soutiennent l'existence du monument. Son édification dans la ville d'Abidjan est également argumentée à la lumière de la compétition politique locale à la fois en termes de ressource et de contrainte dans le renouvellement urbain. Plus loin, par cette posture théorique, le document traite de la caution du monument par le groupe d'acteurs qui s'identifie au travers de lui sous les composantes de " crise », de légitimation de pouvoir, de contrôle de l'espace urbain et d'instrument de lutte identitaire. Ces pratiques susmentionnées sont enfin étudiées sans s'attarder sur les incertitudes qu'elles génèrent et de la réaction des acteurs de sa contestation. L'action organisée comme support de légitimation de pouvoirs autour du «monument des martyrs " de 2002 à 2010 permet dans ce travail de mettre en évidence les échelles organisationnelles suscitées par Gbagbo Laurent et les militants du FPI dans les catégories d'analyses ci-après .

\section{La construction de la valeur historique et culturelle du « monument des martyrs ॥}

A l'image de la ville de Grand Bassam ${ }^{26}$, doter Abidjan d'une telle infrastructure est perçu chez « les militants et fidèles ${ }^{27}$ de Gbagbo Laurent comme une valeur ajoutée au patrimoine culturel de la ville. Ainsi, tout comme à Grand Bassam où « le monument des femmes battantes » permet de rappeler la marche des femmes pour la libération de prisonniers politiques en 1949 (Diabaté, 1975 ), par l'édification d'un monument similaire sur l'espace abidjanais, il s'agit d'inscrire dans l'histoire politique de la ville, le symbole d'une lutte

${ }^{26}$ Ville historique voisine à Abidjan, Grand-Bassam fut la première capitale de la Côte d'Ivoire de 1893 à 1900.

27 Entre 2000 et 2010, la presse écrite proche du RDR désignait ainsi les militants et les sympathisants du FPI. Lire à ce propos Sanga (2003, P.2) 
dite patriotique présentée comme le résultat d'une protestation ayant eu l'adhésion de tous les partis politiques. Expliquant cette situation, Gbagbo Laurent précise dans cette déclaration:

" (...) le peuple était déjà dans la rue, je n'ai fait qu'accompagner le mouvement. Et ceux qui étaient dans la rue, il n'y avait aucun parti, c'étaient tous les partis... » (Extrait du discours recueilli dans Fraternité Matin, 10 novembre 2000, p.4) Même si des tendances à " catégoriser les morts, à opérer une distinction entre les morts » sont activées par Alassane Ouattara et les sympathisants du RDR, pour les militants du FPI, ce monument reste la marque symbolique structurante de la présence des victimes du conflit fratricide lié aux évènements de 2000 et érigées en martyrs (Gbagbo, 2000 ; Tiesse, 2000 ; Ekra, 2014). Alors, au-delà des différences entretenues sur le statut des victimes incarnées dans le monument et promu dans la culture de l'embellissement de la ville, il y aurait des morts dépersonnalisées, c'est-à-dire n'ayant pas « de parti, de religion, de région »(Gbagbo Laurent, 2000). S'inscrivant dans une telle perspective, les militants du FPI ont ainsi proclamé le « monument des martyrs " comme un bien commun avec des caractéristiques de portée nationale.

Pour eux, la légitimation de cette infrastructure culturelle renvoie à la promotion d'un outil du développement urbain. C'est aussi un investissement identitaire par lequel l'image de la ville est rehaussée non seulement par une perspective patrimoniale en matière de monument sculpté mais également, pat les stratégies ciblées en faveur de promotion de ressources dans le champ précité. Il en est de même pour les impératifs de la production de lien social valorisant et d'innovation relative à l'acquisition de symbole identificatoire commun (Basilico, 2005 ; Guerin, 2008). Cette façon de marquer la culture de la ville par la succession de couches de monuments est mise en évidence par cet interviewé :

"le monument rappelait l'histoire de la ville, en prenant appui sur les évènement qui y sont passés(...). Cela montre aussi la culture de la ville et quelque part aussi l'histoire de la Côte d'Ivoire pour les générations futures (...) Chaque régime marque sa trace par une couche de monuments. Sous Houphouët, il y a eu des monuments, sous Bedié également. Sous Le Président Gbagbo, un autre type de monuments a émergé sur la base des évènements qui se sont déroulés. Chaque génération marque son temps par une succession de couches de monuments qui rappelle notre histoire. Il faut les 
conserver au lieu de les perdre. (Entretien avec $\mathrm{Z}$ conseiller au FPI)

Comparant cette manière de faire à la renommée acquise par le « monument des femmes battantes » dans la ville de Grand Bassam, le « monument des martyrs » est devenu la ressource leur permettant de renforcer l'image historique de la « grande » ville qu'est Abidjan. Ensemble, ces deux monuments rappellent que ces villes historiques sont des lieux de mémoire. Et si la première a pu parvenir au statut d'ancienne capitale coloniale patrimonialisée (Guenneguez, 2015), c'est par cette quête d'une identité compétitive au travers du « monument des martyrs » que la seconde peut aussi parvenir à ce statut.

Motivée donc par le besoin de légitimer le « monument des martyrs " dans le patrimoine abidjanais, la décision du Président Gbagbo Laurent d'édifier ledit monument a été transformée en une règle organisationnelle. Par-delà ce fait, l'idée qu'il incarnerait le symbole d'une résistance sociale, le refus de voir se fixer et se cristalliser la compétition politique sur le modèle de la violence ont reconstruit la perception que l'on a de lui au plan culturel. En d'autres termes, il a été brandit par les partisans de Gbagbo Laurent comme un investissement dont la présence serait le symbole de la non-violence prôné par leur parti. Donc dans le champ culturel, sa présence stratégique dans le développement urbain est ainsi perçue par les militants du FPI à la fois comme une forme d'expression de leur identité dans la ville, de captation de l'électorat significatif dont dispose le parti et de la conservation de ses traces mémorielles (Nicolas et Zanetti, 2014)

\section{Le «monument des martyrs » comme symbole de lutte politique et marque de citoyenneté urbaine}

En tant que référence en monuments sculptés dans la ville d'Abidjan, le « monument des martyrs » a donc été investi comme un marqueur de sens à travers lequel les sympathisants de Gbagbo Laurent lui confèrent une image dont les bases ne seraient ni ethniques, ni régionales, ni encore partisanes. Ils justifient ainsi sa présence par le fait qu'il n'a pas été édifié pour servir les intérêts d'un quelconque parti ou groupe ethnique de la scène politique, mais plutôt pour marquer la ville par un instrument touristique qui retrace son identité. Depuis son inauguration en 2002, chez les militants du FPI, ce qui est privilégié dans sa promotion, c'est plutôt sa prise en compte dans un type idéal d'embellissement urbain où l'on peut retracer l'histoire de la lutte historique qui a porté le leader de ce parti au pouvoir. Cette expérience est relatée par cet enquêté qui cite : 
" Le monument était un instrument touristique en rapport avec la ville. Comme beaucoup qui avaient été construits dans les quartiers, par exemple à Adjamé, il y avait le monument Niangui Ambrogoua. Cela donne à la ville un repère. Le régime du Président Gbagbo voulait donner à chaque commune de la ville et à la ville elle-même un repère, un symbole. " (Extrait d'entretien avec Y, membre de la jeunesse du FPI)

Il ressort aussi des réponses de l'enquête que le monument serait dédié « à la vie et non à la mort ». Il pourrait être compris comme un appel à l'espérance dans les droits de la personne (Gneproust, 2002, P. 2). Outre le fait que sa présence sur l'espace urbain abidjanais reste un signe distinctif de l'identité du FPI, il reste aussi une infrastructure par laquelle au cours de la période de crise, " les patriotes $\gg^{28}$ forgent et proclament leur citoyenneté dans la ville (Konaté, 2003 ; Babo, 2005 ; Banegas, 2007, 2010). En revanche, à l'instar de sa présentation comme la base du processus fondateur de l'identité de la ville d'Abidjan au plan politique, ils le reconstruisent singulièrement en un opérateur par lequel ils valorisent l'identité de leur parti, légitiment leur participation à la vie de la ville et/ou de la nation. Ce qui est renseigné dans le verbatim suivant:

"Le monument rappelle un moment de l'histoire des jeunes combattants $d u$ FPI dans l'histoire de la Côte d'Ivoire ... $C$ 'était vraiment un repère des jeunes militants du FPI en ce sens que beaucoup de ces jeunes sont tombés en réclamant la victoire de leur candidat Gbagbo Laurent. Il leur rappelait un temps fort pour leur parti. Il est aussi la marque de la jeunesse qui est sortie braver les tirs de l'armée française (...). Les autorités avaient déposé aussi un gerbe de fleurs devant ce monument en l'honneur de ces victimes...» (Extrait d'entretien avec $\mathrm{G}$, titrologue à Adjamé)

L'analyse de cet extrait d'entretien permet de faire ressortir plusieurs points de similitude entre la mobilisation ou l'automobilisation des « jeunes » du FPI (en tant que groupes stratégiques)

\footnotetext{
${ }^{28} \mathrm{~A}$ l'époque de la crise, notamment entre 2000 et 2010 , les sympathisants et militants du FPI, fidèles à Gbagbo Laurent parce que le reconnaissant comme le Président légitime au pouvoir, s'autodésignaient ainsi afin de mettre en évidence leur attachement à la patrie ou leur zèle à la défendre contre les acteurs probables voulant la déstabiliser. Pour Konaté (2003, P. 63) ils se définiraient comme " un mouvement de résistance ouverte qui soutient le chef de l'État, la Constitution, bref la légalité républicaine que précisément les rebelles remettent en question par leurs revendications de résistance ouverte qui soutient le chef de l'État, la Constitution, bref la légalité républicaine que précisément les rebelles remettent en question par leurs revendications »
} 
et leurs aspirations à s'identifier dans le monument. En effet, dans le travail identitaire mené autour du monument, sont mentionnés la lutte des « jeunes » du parti et les liens incités entre les réseaux de résistance qu'ils ont mis en place. Les modes d'intervention et de coordination pour la défense de leur parti, de la ville d'Abidjan ou $\mathrm{du}$ pays sont aussi prises en compte. La particularité de cette construction identitaire, c'est que l'identification de ces « jeunes » dans le monument est valorisée à travers leur participation aux évènements politiques (notamment par les marches de contestations) de la ville. De tels évènements, associés à la gouvernance urbaine de l'époque servent alors de vecteur directeur permettant d'ajuster l'identité de martyrs à celle de la ville, et par-delà ce fait, revendiquer la reconnaissance institutionnelle de ces «jeunes » engagés pour la défense de l'Etat (Gravari-Barbas et Jacquot, 2007). Cette manière de construire 1'identité de la ville d'Abidjan par la mobilisation des évènements politiques qui s'y sont produits est valorisée par cet interviewé dans ces propos : " (...) il s'agissait de refaire l'histoire de la ville, lui donner une nouvelle identité en prenant appui sur les évènements de la crise politique comme symboles...Alors pourquoi certains hommes refusent qu'on retrace cette histoire de la ville de cette manière-là ? Cela montre aussi la culture de la ville! Alors, pourquoi préférer des jets d'eau à la place de ce monument qui retrace en partie une histoire de la ville? " (Extrait d'entretien avec Y, membre de la jeunesse du FPI)

Aussi, par le transfert de sens, l'identité des « jeunes combattants » est-elle brandie comme le support de cristallisation et de diffusion des valeurs collectives qu'incarnent la lutte de résistance menée par Gbagbo Laurent et son parti. Par le travail de marquage identitaire opéré à travers celle-ci, ces acteurs sont plutôt devenus un capital reconnu et valorisé au plan national par l'Etat. Ils lui servent d'appui pour concilier l'histoire de de la participation à la vie du parti au pouvoir à celle de l'intégration ou la citoyenneté dans la ville, voire $\mathrm{du}$ pays. En combinant ces trois histoires, les militants du FPI tentent de se réapproprier la mémoire cette entité territoriale . Ce qui serait même renforcé par les décisions informelles prises au niveau de l'Etat et qui concourent à valoriser l'engagement de ces « jeunes » à la défense de la ville. Le plus important, c'est d'arriver à consolider l'identité de dernière, identité où se mêlent divers éléments stratégiques tels que les volontés de revendication d'autonomie vis-à-vis de l'excolonisateur, du renforcement de la légitimité du pouvoir. Le parti au pouvoir procédaient ainsi pour l'uniformisation de son identité politique à 
travers les logiques de recompositions spatiales, les fabriques de cohésion et de solidarités urbaines (Hommage, 2006 ; Ginet 2014).

FPI ont permis de déboucher sur des formes de reconnaissance associées à la présence du monument dans la ville. Ils ont aussi permis de créer son lien avec les revendications collectives au sein de la ville. Par les processus en question, leurs valeurs politiques et leurs tentatives de légitimation d'une identité nationale ont été mises en relief dans la résistance sociale. En somme, sur la base des expérience relatives à la participation aux évènements politiques de la ville, l'identité de ces « jeunes» se construit à travers les actions collectives. C'est dans une telle perspective que l'honneur rendu aux « jeunes combattants » du FPI fonctionnerait également comme étant contre l'exclusion des « jeunes » des partis rivaux qui ne s'identifient pas dans le monument. Cet hommage, au risque de produire des types de stigmatisation et de mépris passe ainsi par l'effacement de l'identité des premiers jeunes susmentionnés. Par cette façon de faire, l'objectif serait de maintenir une image valorisante de ces « martyrs de la nation », contrairement à ceux réclamés par l'adversaire politique qu'est le RDR. Cette production de l'identité urbaine par la marque symbolique de la résistance et de la participation des «jeunes» du FPI ou du pays sont les traits caractéristiques par lesquels l'identité politique de la ville se construit.

\section{Le sens politique de la présence du « monument des martyrs » dans la ville d'Abidjan}

$\mathrm{Au}$ plan politique, les données de l'enquête permettent de souligner que les identités construites autour du monument sont surtout des identités engagées. Ces dernières contribueraient premièrement à dénoncer la violence politique traversée dans le pays en privilégiant le lien entre le monument et le contexte de son édification. Pour ce faire, chez les militants du FPI, la symbolique du monument ne se limite pas seulement à la condamnation de la crise fratricide qui l'a fait naitre. Sa présence est bien plus qu'un impératif pour la quête de réconciliation et les démarches de reconsolidation de la cohésion sociale. Selon eux, ce monument devrait servir à rompre avec les formes évolutives de la violence politique en Côte d'Ivoire (Akindes, 2008; Vidal, 2008). C'est ce point de vue que confirme d'ailleurs Gbato (2002, P.2) lorsqu'il relate ces propos de Séri Bailly ${ }^{29}$ :

29 Acteur de premier rang du FPI, Séri Bailly était le Ministre de l'Enseignement Supérieur et de la recherche Scientifique à l'époque de la cérémonie d'inauguration $\mathrm{du}$ monument. Il avait eu la charge de présenter au nom du gouvernement les significations susceptibles d'être dégagées de la symbolique du monument. 
"Le monument de la place des martyrs est une parole que notre peuple s'adresse à lui-même pour dire non à la violence politique. Afin qu'instruits des expériences du passé, les Ivoiriens agissent de sorte que, plus jamais, ce qui s'est passé en octobre 2000 ne se répète en Côte d'Ivoire "

Le monument, au-delà d'être un instrument d'identité politique est aussi mobilisé pour se défendre contre les pratiques déconstructives des partis rivaux (Vanderlick et Batungwanayo, 2012). Il s'agit notamment des controverses et des récits non officiels, les préjugés ou stéréotypes favorables à sa disqualification de l'histoire de la ville. En effet, motivés par l'idéologie du devoir de mémoire envers lesdites victimes, les militants du FPI perçoivent sa promotion comme l'appareil productif par lequel ils témoignent d'une part leur attachement au nationalisme. Prenant appui sur la même idéologie, ils tentent d'autre part de rendre officiels leurs récits relatifs aux expressions de la violence politique dans la ville et/ou dans le pays.

A l'examen de l'extrait de discours ci-dessus, les données de l'enquête révèlent également que la commémoration du monument a été perçue par les militants du RDR comme un outil sur lequel les militants du FPI s'appuient pour légitimer la domination de leurs adversaires politiques. Par une telle croyance, il a contribué à mettre en place des perspectives de solidarité collective allant dans le sens de la défense de la ville ou du pays " attaqué ». En d'autres termes, au plan politique, les objectifs visés par les formes de production identitaire autour du monument seraient surtout les enjeux de pouvoirs (Nossent, 2014). En passant par les pratiques de l'enracinement politique de leur modèle de gouvernance au sein de la ville d'Abidjan, la manifestation de ces enjeux de pouvoirs a contribué à renforcer la légitimité de l'État, à déconstruire l'idée de sa fragilité et à reconstruire la confiance en la lutte collective que mènerait le Président Gbagbo Laurent et les sympathisants du FPI. C'est surtout par la production et la pérennisation desdits enjeux de pouvoirs autour du monument qu'à travers ledit président, leur leader politique au pouvoir, les militants du FPI ont pu affirmer l'autonomie de leur parti au cours de la période de crise (2002-2010). Ils en usent également pour faire montre de leurs compétences à s'adapter aux transformations politiques structurantes (création de coalition politique par exemple) à l'œuvre dans le pays.

\section{Le « monument des martyrs » et les institutions urbaine et/ou nationale}

L'étude montre qu'en comparaison avec la ville de Grand Bassam, Abidjan a en commun avec celle-ci l'expérience des luttes 
patriotiques menées contre les pratiques d'impérialisme et de néocolonialisme français en Côte d'Ivoire (Banegas, 2006 ; Akindes et als, 2010). A partir donc de deux conjonctures sociales avec plus ou moins des traditions politiques similaires, ces symboles traduisent pour Gbagbo Laurent et les sympathisants du FPI la volonté de revendiquer à la fois le droit d'être autonome et de rompre avec les pratiques d'injustice. Même s'ils ont été érigés dans des contextes politiques plus ou moins différents (colonisation et multipartisme postindépendance), l'un ou l'autre de ces symboles témoignent de la reconnaissance et de la légitimation d'un pouvoir. Pour ce qui est du «monument des martyrs », allusion est faite à la deuxième République. C'est à juste titre que le Président Gbagbo Laurent (2000) scande :

" (...) la deuxième République nait dans la grande douleur. Une naissance qui rappelle la naissance de la première République. Souvenons-nous des morts de Dimbokro, (...) de Grand Bassam (...) Hier le peuple épris de liberté affrontait la violence coloniale, les victimes étaient de toutes les régions, étaient de différentes confessions mais unies contre un système qu'ils reprouvaient... Aujourd'hui c'est dans un affrontement fratricide que le peuple de Côte d'Ivoire a payé le prix... » (Extrait de discours dans Fraternité Matin du 10 novembre 2000, P.4)

Cet extrait de discours révèle surtout que l'édification du monument est justifiée par la volonté de matérialiser une forme d'affirmation collective et singulière du pays. Il y apparait des non-dits à travers lesquels la dénonciation du conflit interne par la symbolique $\mathrm{du}$ monument est mise en relation avec la déconstruction des rapports de force en faveur de Gbagbo Laurent. Si les sympathisants de ce leader sont assimilés au « peuple », c'est en vue de fabriquer la cohésion autour de sa victoire électorale sous les formes de solidarité, de justice et de protection sociale. Le proclamant, ce sont plutôt ces valeurs qui sont généralement reconstruites comme attentes vis-à-vis de sa gestion du pouvoir. Elles seraient aussi la ressource par laquelle les militants du FPI fondent et légitiment son statut de chef d'Etat.

Dans la valorisation des paramètres institutionnels de la ville, le monument représente un objet de conquête sociale. Son édification et sa promotion sont confondues à la volonté du « peuple » ivoirien et non celle d'un parti politique. Il est même assimilé à un attribut du pouvoir laissant apparaitre les sens d'égalité, de protection sociale, de solidarité et de justice sociale. Une telle innovation au plan institutionnelle laisse surtout entrevoir que la singularité du monument s'inscrit au centre de la gouvernance de la ville. La comparant aux villes historiques du 
pays en matière de résistance et de construction de la nation, sa présence est mise en valeur comme une pratique productive. Par elle, de nouveaux types de liens «forts » sont encouragés dans le sens de la polarisation d'une identité nationale valorisante. Au plan institutionnel, l'effort consenti par les victimes, ces nouveaux « héros » de la nation et les événements desquelles découle l'édification du monument renforcent l'identité de la ville comme lieu historique pour les contemporains (Osborne, 2001).

En ne les faisant pas passer sous silence, ils sont de ce fait inclus dans la mémoire collective de la ville et du pays. Ce qui permet aux sympathisants du FPI d'innover en matière d'influence de l'identité de leur parti. Les ressources idéologiques de cette innovation restent essentiellement les croyances en la lutte contre l'exclusion sociale. Il ya aussi l'idée selon laquelle au travers des institutions urbaines, le monument doit être représentatif par sa perception à l'antipode des affirmations de discrimination vécues ou rencontrées, des plaintes de marginalisation et de violences réelles ou supposées. Ces pratiques, quitte à contribuer au positionnement des militants de partis qui contestent le monument en victimes (le RDR surtout), et polariser leur doctrine de ségrégation politique ${ }^{30}$ sont ainsi dissuadées dans la manipulation stratégique des règles officielles ou non relatives à sa valorisation. L'enjeu serait de rendre une telle doctrine impopulaire et sans valeur à l'échelle nationale. Compte tenu de ce fait, Gbagbo Laurent et les sympathisants du FPI défendaient l'opinion selon laquelle le monument en question ne doit sous aucun prétexte être la preuve conséquente d'un marqueur dévalorisant au sein de la ville. Posture d'ailleurs susceptible d'être favorisée et instrumentalisée par les mobilisations ethniques partisanes qui découlent de la compétition politique en vogue à cette l'époque.

\section{La présence du « monument des martyrs » et le renouvellement urbain}

Du corpus de données recueillies, il ressort de même que le choix du site sur lequel le monument a été construit reste un facteur clé dans l'aménagement de l'espace au plan politique. En effet, dépassant la seule volonté d'honorer lesdits martyrs, « la place des martyrs »se veut aussi la réponse à une approche stratégique de renouvellement urbain. Essentiellement aménagé pour produire un imaginaire de solidarité ou de cohésion urbaine, chez les militants du FPI, le site du monument est perçu comme un espace susceptible de recréer également des liens valorisants. Cela se décline sous trois aspects : au plan

\footnotetext{
${ }^{30}$ Allusion est faite aux types de persécutions, menaces, les actes de barbaries dont les militants de ce parti auraient été l'objet.
} 
idéologique, la présence du monument sur un site découvert de la ville vise à déconstruire une image de celle-ci diffusée dans les media internationaux comme étant au bord de l'implosion (Roubaud, 2003). Il s'agit, dans cet ordre d'idées, d'associer l'identité de la ville d'abidjan à une dimension humanisante. Ce qui dénote des propriétés idéologiques véhiculées au travers du monument. Ce sont entre autres, les idées de triomphe de la liberté, de réconciliation nationale et de rupture avec la violence politique. Il en est de même pour les croyances selon lesquelles la ville serait devenue un espace sociale où se distinguent les valeur de promotion des droits humains, la célébration de la démocratie et la production d'un nationalisme singulier dans la sous-région.

$\mathrm{Au}$ plan symbolique, la position géographique du monument ne se veut pas anodine. Afin de renforcer sa marque de notoriété dans l'aménagement de la ville d'Abidjan, le régime Gbagbo, à travers le Ministère de la construction et de l'urbanisme de l'époque, l'a " implanté à un endroit symbolique: entre un quartier chic, Cocody, et un quartier populaire, Adjamé " (Opeli, 2002, P.2). A partir d'une telle remarque, il ne serait pas faux de soutenir que pour Gbagbo Laurent et les militants du FPI, l'aménagement du site occupé par le " monument des martyrs » est voulu comme étant en phase avec la recherche de la cohésion urbaine. Les caractéristiques majeures de cette quête de cohésion sont d'une part, l'effacement des frontières spatiales et identitaires de la ville. Le but recherché est donc d'y produire et consolider une identité collective valorisante. Cette dernière devrait surtout conférer au monument les valeurs d'intégration, d'unité et de solidarité nationale. Par conséquent, tout comme les victimes honorées n'ont « pas de parti, de religion, de région » (Gbagbo, 2000) le choix $\mathrm{du}$ site sur lequel le monument est édifié serait fait avec neutralité .

Au plan structurel, affiché dans la neutralité aussi bien ethnique, politique que spatial, il convient de dire que pour Gbagbo Laurent et les militants du FPI, le monument ne doit pas être l'expression d'une barrière sociale. Bien plus, au niveau urbain et/ou national, il ne doit laisser l'image d'un moyen de différenciation politique, de division culturelle et d'effritement des appartenances sociales. Il doit être au contraire un instrument d'intégration et de renforcement de liens urbains. Sa position frontalière est alors vantée comme une innovation dans l'aménagement de la ville (Raffestin, 1986 ; Amilhat Szary, 2011). Elle vise surtout la consolidation d'une identité collective fabriquée sur la base de ses propriétés symboliques citées plus haut. Dans le même sens, le FPI et ses sympathisants l'affichent comme étant en déphasage non seulement avec les frontières réelles entre les partis politiques implantés dans la ville mais aussi, avec ses limites administratives. Le monument 
contribuerait en ce sens à modifier l'identité de la ville au niveau politique, la faisant plus ou moins évoluer vers la reproduction des attentes de l'unité nationale.

Par de telles stratégies, surtout au cours de la période de crise (2002 à 2010), les propriétés symboliques du monument en tant que marques porteuses de sens et de significations ont été conférées à la ville d'Abidjan. Par elles, les désignations suivantes lui ont été associées afin de consolider ses appellations ou ses identités de ville martyre, de résistance et d'innovation en matière de production d'identité collective.

\section{Discussions et conclusion}

En décomposant les pratiques relevées dans ce travail comme les parties constitutives de la légitimité politique construite sous le règne $\mathrm{du}$ Président Gbagbo Laurent, diverses illustrations ont été analysées. L'usage du monument y parait pertinent pour décrire les processus allant dans le sens de gestion de situations conflictuelles et de la construction de cohésion sociale. Du point de vue épistémique donc, le travail présente trois intérêts majeurs.

Premièrement, s'inscrivant dans la perspective de consolidation d'une légitimité politique, la promesse de l'édification du monument se dégage comme une norme d'action publique. C'est cette promesse qui amène à délimiter son usage afin de le distinguer et le légitimer dans l'ensemble urbain. En tant que ressource mobilisable, par cette norme, le monument a été exploité dans un contexte d'interaction conflictuelle. Pour ce faire, il a été perçu dans les rivalités politiques avec le RDR par exemple comme le signe d'une stratégie politique à l'usage d'un groupe dominant : le FPI, parti au pouvoir. Chez les sympathisants du RDR, par cette position de parti au pouvoir, le FPI aurait tenté de légitimer sa domination par cet instrument symbolique qu'est le monument. Mais, les zones d'incertitudes liées à cette tentative de légitimation de pouvoir vis-à-vis de ses concurrents politiques ont été de façon inverse mobilisées par ces derniers pour légitimer leur vulnérabilité. Dans la présente étude, en tant que politique publique organisée, l'organisation de la légitimité politique en système structurant permet de révéler la convergence de tels résultats avec les travaux sur l'importance et la complexité des politiques publiques mémorielles (Balayn , 2013 ; Vinyes, 2013).

Deuxièmement, en prenant appui sur les pratiques incitées autour du «monument des martyrs", il ressort de cette étude qu'il est une ressource à la fois culturelle, politique et symbolique. Il sert ainsi de base à la production de diverses expressions de citoyenneté, la génération d'un idéal-type de gouvernance spatiale. Le monument 
demeure ainsi dans la ville, le témoignage d'une ressource valorisée autour de laquelle sont activées les pratiques de participation et d'appartenance à la ville, les formes de conflits non manifestes. Sa présence influence les tendances de consolidation ou de fragilisation de la cohésion sociale, la reconstruction de frontières identitaires et la reconstitution des identités collectives. Au regard de tels résultats, cette étude se veut aussi convergente avec les conclusions de celles conduites par Petithomme (2015) et Fontaine (2016).

Troisièmement, les conclusions de cette étude permettent de comprendre que l'aménagement urbain peut être productif par la diversité des évènements qui s'y produisent. En tant que cadre des expériences identitaires, il permet de mettre en évidence le renouvellement de formes spatiales en rapport avec le contexte historique, les idéologies de réinvention de la ville et les investissements en matière d'infrastructures qui la consolident. Le «monument des martyrs» comme un investissement identitaire dans la ville en est un exemple. Edifié à la mesure du nationalisme, sa présence ouvre des perspectives nouvelles dans le fonctionnement de la cohésion urbaine et les dispositifs mis en place pour la gouvernance de la ville. Au travers des différentes perceptions qui ont marqué la réflexion urbaine au cours de la période de crise, c'est-à-dire entre 2002 et 2010, la présence du monument a fait varier les représentations liées à l'identité de la ville, la restructuration de son espace au plan politique et les production d'identités collectives en son sein. Dans cette approche, l'étude rejoint les analyses de Ginet et Wiesztort (2014).

Sur la base des pratiques suscitées par ses promoteurs, le « monument des martyrs » a donc été le symbole de la résistance urbaine, un facteur d'intégration et de cohésion urbaine. En tant qu'investissement de légitimité politique, il n'a plutôt que servi à renforcer la radicalisation des identités et des enjeux suscités autour de lui. Pour ces mêmes raisons, son attractivité a été en conséquence limitée. Il serait alors opportun de pousser la réflexion vers la définition de critères objectifs quant à la dotation de la ville d'Abidjan de tel patrimoine culturel c'est-à-dire de monuments sculptés.

\section{References:}

1. AKINDES, F. (2008). Racines des crises socio-politiques en Côte d'Ivoire et sens de l'histoire. In Frontières de la citoyenneté et violence politique en Côte d'Ivoire. Sous la direction de Jean Bernard Ouédraogo et Ebrima Sall. Dakar, CODESRIA. Consulté le 12 Septembre 2016. Disponible sur http://www.codesria.org/spip.php?article1393 
2. AKINDES, F., Moussa, F. \& Gnangadjomon, K. (2010). Côte d'Ivoire : insurrection et contre-insurrection. In Alternatives Sud, vol. 17, $\mathrm{n}^{\circ}$ 93, p 93-97. Etat des résistances dans le Sud Afrique. Consulté le 12 Septembre 2016. Disponible sur http://www.cetri.be/cote-d-ivoire-insurrection-e

3. ALBERT, J-P. (2007). Martyre et mort volontaire en Europe. : Entre religion et politique. In Bulletin of Death and Life Studies, vol III, La mort et les au dela", 21st Century COE Program DALS, The University of Tokyo, pp.79-91. Consulté le 10 Décembre 2016 Disponible sur https://hal.inria.fr/docs/00/36/99/34/PDF/Martyre_et_mort_volontair e.pdf

4. AMILHAT SZARY, A-L. (2011). Identités collectives à la frontière, Civilisations .60-1 | Consulté le 16 juillet 2017. Disponible sur http://civilisations.revues.org

5. ARBORIO, A-M. (2007). L'observation directe en sociologie : quelques réflexions méthodologiques à propos de travaux de recherches sur le terrain hospitalier. In Recherche en soins infirmiers vol 3, $\mathrm{n}^{\circ}$ 90, p 26-34. Disponible sur http://www.cairn.info/revue-recherche-en-soins-infirmiers-2007-3page-26.htm

6. ASSOUMAN, B. (2015). La réconciliation, un choix imposé. In Penser la réconciliation pour panser la Côte d'Ivoire, p 73-91. L'Harmattan

7. AUBRY, M. \& MATTHIEU DE OLIVEIRA, (2014). « Une base de données sur les monuments aux morts : histoire concrète et valorisation numérique », In Situ, 25. Consulté le 30 septembre 2016. Disponible sur http://insitu.revues.org/

8. BABO, A. (2005). Citoyenneté et jeu politique en Côte d'Ivoire. In Kasa bya Kasa, $\mathrm{n}^{\circ}$ 8, p. 23-37.

9. BALAYN, E. (2013).Vers une européanisation du secteur des politiques publiques mémorielles. In Sens Public, Revue internationale International Web Journal. http://www.senspublic.org/article1003.html

10. BANEGAS, R. (2006). Côte d'Ivoire : une guerre de la seconde indépendance? Refonder la coopération française sur les brisées du legs colonial. In FASOPO, p 197-251.Consulté le 11 Octobre $2016 \quad$ Disponible sur http://www.fasopo.org/publication

11. BANEGAS, R. (2007). Côte d'Ivoire : les jeunes « se lèvent en hommes ». Anticolonialisme et ultranationalis chez les Jeunes patriotes d'Abidjan. In Les études du $C E R I, \mathrm{n}^{\circ} 137, \mathrm{p}$ 
1-52. Consulté le 11 Octobre 2016. Disponible sur http://www.sciencespo.fr/ceri/sites/sciencespo.fr.ceri/files/etude137.p df.

12. BANEGAS, R. (2010). La politique du " gbonhi ». Mobilisations patriotiques, violence milicienne et carrières militantes en Côte-d'Ivoire. In Genèses /4, $\mathrm{n}^{\circ}$ 81, p. 25-44. Consulté le 11 Octobre 2016.Disponible sur https://www.cairn.info/revue-geneses-2010-4-page-25.htm

13. BASILICO, S. (2005). Redéfinir le Patrimoine culturel à l'heure de la globalisation. In Des cultures et des Hommes. Clefs anthropologiques pour la mondialisation, L'harmattan, Collection Logiques sociales, pp.15. Disponible sur https://archivesic.ccsd.cnrs.fr/sic

14. BOUMAZA, M. \& CAMPANA, A. (2015). Comment fabriquet-on des martyrs? Enjeux théoriques et méthodologiques. Introduction. In Signes, Discours et Sociétés, La fabrique des martyrs. Consulté le 10 Décembre 2016. Disponible sur http://www.revue-signes.info/document.php?id=4632.

15. BRUNEAU, M. \& PAPOULIDIS, K. (2003). La mémoire des « patries inoubliables ». La construction de monuments par les réfugiés d'Asie mineure en Grèce, Vingtième Siècle. Revue d'histoire 2003/2 (no 78), p. 35-57 Disponible sur https://www.cairn.info/revue-vingtieme-siecle-revue-d-histoire-20032-page-35.htm

16. CHAPOULIE, J-M. (2000). Le travail de terrain, l'observation des actions et des interactions, et la sociologie. In Sociétés contemporaines $\quad \mathrm{n}^{\circ} \quad 40, \mathrm{p}$ 5-27. Disponible sur http://www.persee.fr/doc/socco_1150-1944_2000_num_40_1_1811.

17. CHOAY, F. (1992). L'Allégorie du patrimoine. Paris : Seuil, 273 $\mathrm{p}$

18. COTE, L. \& TURGEON, J. (2002). Comment lire de façon critique les articles de recherche qualitative en médecine. In Pédagogie Médicale, vol 3, p 81-90 Disponible sur http// :www.uqtr.uquebec.ca/metho-lcs/DOC/grille_cote-turgeon.pdf

19. CROZIER, M. \& Friedberg, E. (1977). L'acteur et le système, Paris, Le Seuil

20. DIABATE, H. (1975). La Marche des femmes sur GrandBassam. Abidjan; Dakar, Les Nouvelles Editions Africaines, 64p

21. EKRA, F. (2014). Abidjan-Chantiers : topographie d'une scène panafricaine. In Art Press, $\mathrm{n}^{\circ}$ 402, p 48-53. Consulté le 16 Juin 2016. Disponible sur http://cecilefakhoury.com/wpcontent/uploads/2014/10/Abidjan-chantiers.pdf 
22. FONTAINE, M. (2016). " Mourir en martyr » dans le journal : enjeux d'écriture et de pouvoirs. In Effeuillage, la revue qui met les médias à nu $\mathrm{n}^{\circ} 5$, Les feuillets, le supplément numérique. Disponible sur http://feuillets2016.effeuillage-larevue.fr/

23. FRIEDBERG, E. (1993). Le Pouvoir et la Règle. Dynamiques de l'action organisée, Paris, Le Seuil

24. GBAGBO, L. (2000). La mort n'a pas de parti, de religion, de région. In Fraternité Matin, $\mathrm{n}^{\circ} 10000$, du 10 novembre

25. GBATO, G. (2002). Inauguration de la place des martyrs : les héros d'octobre 2000 élevés au panthéon de l'histoire. In Notre Voie, $\mathrm{n}^{\circ} 1099$ du 25 janvier

26. GENSBURGER, S. \& LAVABRE, M-C. (2012). D'une « mémoire 》 européenne à l'européanisation de la " mémoire ", In Politique européenne $/ 2 \quad\left(\mathrm{n}^{\circ}\right.$ 37), p. 9-17. $\mathrm{http} / / / \mathrm{www} . c a i r n . i n f o / r e v u e-p o l i t i q u e-e u r o p e e n n e-2012-2$-page9.htm

27. GHIGLIONE, R., LANDRE, A., BROMBERG, M. \& MOLETTE, P. (1998). L'analyse automatique des contenus. Paris, Dunod.

28. GINET, P. (2014). Le lien défense-territoires. Quelles perspectives pour le Géographe sur le plan scientifique et dans sa pratique de l'aménagement ? Master. Défense, risque, sécurité et aménagement, Metz (Universit_e de Lorraine), France, pp.17. Consulté le 13 juin $201 \overline{7}$ Disponible sur https://cel.archives-ouvertes.fr/cel-00966048

29. GINET, P. \& WIESZTORT, L. (2013). La place de la mémoire dans les aménagements territoriaux, un enjeu géopolitique. , Revue Géographique de l'Est, vol. 53 / 3-4 | Consulté le 02 Octobre 2016. Disponible sur http://rge.revues.org/5059

30. GNEPROUST, K. (2002). Inauguration du mémorial des martyrs : un appel à la réconciliation. In Fraternité Matin, $\mathrm{n}^{\circ} 11168 \mathrm{du}$ 25 janvier

31. GRAVARI-BARBAS, M. \& JACQUOT, S. (2007). L'événement, outil de légitimation de projets urbains : l'instrumentalisation des espaces et des temporalités événementiels à Lille et Gênes ", In Géocarrefour, Vol. 82/3. Consulté le 10 juillet 2017.Disponible sur http://geocarrefour.revues.org

32. GUENNEGUEZ, A. (2015). Le processus d'appropriation symbolique d'une ancienne capitale coloniale patrimonialisée : cas de la "ville historique de Grand-Bassam " en Côte 
d'Ivoire. Mémoire de Recherche en Etudes Africaines, Disponible sur https://openaccess.leidenuniv.nl/bitstream/handle/1887/35171/Mon\% 20memoire.pdf? sequence $=1$

33. GUERIN, M-A. (2008) Le patrimoine culturel, instrument de la stratégie de légitimation de l'Union européenne. L'exemple des programmes Interreg, In Politique européenne $/ 2$ ( $\left.\mathrm{n}^{\circ} 25\right)$, $\mathrm{p}$. 231-251. Disponible sur http://www.cairn.info/revue-politiqueeuropeenne-2008-2-page-231.htm

34. HALBWACHS, M. (1994). Les cadres sociaux de la mémoire, Paris, Albin Michel

35. HAEGEL, F \& LAVABRE, M-C. (2010). Destins ordinaires. Identité singulière et mémoire partagée, Presses de Sciences Po. Disponible sur https://temporalites.revues.org/1277

36. HOMMAGE, C. (2006). Cohérence territoriale et cohésion sociale dans les projets de territoire. Communication présentée au Congrès "Action sociale et territoires », CNAM, Paris. Consulté le 02 mai 2017. Disponible sur http://www.irtsaquitaine.fr/recherche/publications/C_Hommage_cna m.pdf

37. HOUEDIN, B. \& N'GUESSAN, D. (2017). Les monuments d'art de la ville d'Abidjan : de l'embellissement urbain à la lutte politique (2003-2013). In Revue des Etudes Multidisciplinaires en Sciences Economiques et Sociales (REMSES), $\quad \mathrm{n}^{\circ} 5 . \quad$ Disponible sur http://revues.imist.ma/index.php?journal=REMSES.

38. IBRAHIMA, D. (2017). Violence syndicale estudiantine dans les universités publiques de Côte d'Ivoire : perceptions et enjeux. In European Scientific Journal, Vol.13, No.7. Disponible sur http://eujournal.org/index.php/esj/article/download/8981/8546

39. JAGOREL, Q. (2014). Les monuments aux morts, puissant outil mémoriel après la Grande Guerre Disponible sur http://www.lemonde.fr/idees/article/2014/09/22/les-monuments-auxmorts-puissant-outil-memoriel-apres-la-grande guerre_4492159_3232.html\#5c73siTbSosiVqX4.99

40. KONAN, D. (2015). Rénovation urbaine et destruction des monuments d'arts dans la ville d'Abidjan : cas $d u$ " " monument des martyrs " $"$ à Adjamé et de la statue de la liberté à Yopougon. Mémoire de Master 1, pp1-61.Institut d'Ethnosociologie, 2015, Université Félix Houphouët Boigny, Abidjan 
41. KONATE, Y. (2003). Les enfants de la balle. De la Fesci aux mouvements de patriote. Politique africaine, N89. pp49-70.

42. KOULIBALY, M. (2003). La guerre de la France contre la Côte d'Ivoire. l'Harmattan, Paris

43. LIA, J-S. (2001).Les boulevards de l'université et des martyrs ouverts à la circulation, in Notre Voie, $\mathrm{n}^{\circ} 10767$ des samedi 29 et dimanche 30 décembre

44. MUCCHIELLI, R. (2006). L'analyse de contenu des documents et des communications. Issy-Les Moulineaux : ESF (9ème édition).

45. NICOLAS, A. \& ZANETTI, T. (2014).Usages de la mémoire dans les projets de renouvellement urbain. Le cas des espaces hérités de l'industrie française, In Articulo - Journal of Urban Research, Consulté le 11 Juilet 2017. Disponible sur http://articulo.revues.org/2464

46. NOSSENT, J. (2014). Les commémorations de la Première Guerre mondiale en tant qu'enjeu de pouvoir : étude du plan d'action "Commémorer 14-18. Mémoire présenté en vue de l'obtention du Master en sciences politiques, administration publique. Université de Liège. Consulté le 15 janvier 2017. Disponible sur http://hdl.handle.net/2268/182520

47. OPELI, D. (2002). La symbolique de la liberté et de la paix. In Notre Voie, $\mathrm{n}^{\circ} 1099$, du 25 Janvier

48. OSBORNE, Brian. (2001). Paysages, mémoire, monuments et commémoration : L'identité à sa place. Disponible sur http://canada.metropolis.net/events/ethnocultural/publications/putinde n_f.pdf

49. PEPE, M. (2000). Hommage aux martyrs de la démocratie. In Fraternité Matin, $\mathrm{n}^{\circ} 10000$, du 10 novembre

50. PETITHOMME, M. (2015). Les martyrs comme ressources culturelles des mobilisations : Les cultes de Txiki, Otaegi et Argala par le nationalisme basque radical. In Signes, Discours et Sociétés,La fabrique des martyrs. Consulté le 10 Décembre 2016. Disponible sur http://www.revuesignes.info/document.php?id=4425

51. PIRES, A. (1997). Échantillonnage et recherche qualitative : essai théorique et méthodologique. In Dans J. Poupart, J.-P. Deslauriers, L.- H. Groulx, A. Laperrière, P. Mayer \& A.P. Pirès, La recherche qualitative : Enjeux épistémologiques et méthodologiques, pp.113- 172. Boucherville : G. Morin. Disponible 
http://classiques.uqac.ca/contemporains/pires_alvaro/echantillonnage _recherche_qualitative/echantillon.

52. PREMAT, C. (2010). L'inscription politique des récits mémoriels en France : Lecture de Gouverner les mémoires. Les politiques mémorielles en France de Johann Michel. In Sens Public, Revue internationale International Web Journal. www.senspublic.org

53. RAFFESTIN, Claude. (1986). Eléments pour une théorie de la frontière. In Diogène, vol. 34, no.134, p. 3-21. Disponible sur https://archive-ouverte.unige.ch/unige: 4348

54. ROUBAUD, F. (2003). La crise vue d'en bas à Abidjan : ethnicité, gouvernance et démocratie. In Afrique contemporaine vol 2 n ${ }^{\circ}$ 206, p. 57-86. Disponible sur http://www.cairn.info/revue-afrique-contemporaine-2003-2-page57.htm

55. SANDLAR, C. (2005). " Les « titrologues » de l'ivoirité », In Outre-Terre $/ 2$ (no 11), p. 229-240. Disponible sur http://www.cairn.info/revue-outre-terre1-2005-2-page-229.htm

56. SANGA, C. (2003). Formation des milices, manipulation de la rue...Voici comment Le Président Gbagbo veut ressembler à Hitler. In Le Patriote, $\mathrm{n}^{\circ} 1256$ du 07 novembre

57. SIVORI, B. (2000). Le Président Gbagbo Laurent : « honneurs à ceux qui sont tombés en combattants les mains nues sans haines ».In Notre Voie, $\mathrm{n}^{\circ} 745$ du 10 novembre

58. TEHO, F. et GBAPO, S. (2002). Pierre Djédji Amondji (tète de FPI-PDCI-PIT) : " Tout le monde sait que j'ai fait mes preuves ». In Notre Voie, $\mathrm{n}^{\circ} 1231 \mathrm{du}$ vendredi 05 juillet.

59. TIESSE, C. (2000). Morts pour que vive la liberté. In Fraternité Matin, $\mathrm{n}^{\circ} 10000$, du 10 novembre

60. VANDERLICK, B. \& BATUNGWANAYO, A. (2012). Les lieux de mémoire, initiatives commémoratives et mémorielles du conflit burundais: Souvenirs invisibles et permanents. In Perspectives Series: Research Report. Disponible sur http://www.impunitywatch.org/docs/Burundi_Mem_Research_Repor t_FR.pdf

61. VIDAL, C. (2008). La brutalisation du champ politique ivoirien, 1990-2003. In Frontières de la citoyenneté et violence politique en Côte d'Ivoire. Sous la direction de Jean Bernard Ouédraogo et Ebrima Sall. Dakar, CODESRIA, Consulté le 12 Septembre 2016 Disponible sur http://www.codesria.org/spip.php?article1393

62. VINYES, R. (2013). Le processus de construction d'une mémoire publique par l'État espagnol, In Témoigner. Entre 
histoire et mémoire. Consulté le 11 juillet 2017. Disponible sur http://temoigner.revues.org/445 